\title{
HISTORIA URBANA Y URBANIZACIÓN EN LA ESPAÑA MODERNA
}

por

DAVID R. RINGROSE

University of California, San Diego.

RESUMEN: Este artículo parte de las argumentaciones presentadas por Pablo Sáncheq León en su contribucion a este número de Hipania para reflexionar sobre las relaciones entre fuerzas de mercado y factores institucionales en la evolución de las pautas de urbanización de la Península Ibérica entre los siglos XVI y XIX. $E$ l profesor Ringrose distingue diversas regiones dentro de la Peninsula con dinámicas y redes urbanas caracteristicas, revisa las interpretaciones dominantes sobre los efectos de la crisis del siglo XVII en las pautas de urbanización y prolonga basta finales del siglo XVTII e incluso después algunos de los rasgos característicos de las pautas y las redes de urbanización de esta región de la Europa del Sur.

PAlabras Clave: Urbanización. Ređ̛ urbana. Castilla. Edad Moderna. Mercados. Instituciones. Racionalidad económica.

ABSTRACT: This article takes as a starting point the arguments presented by Pablo Sancheq Leon in his contribution to this issue of Hispania in order to reflect on the relationsbips between market forces and institutional factors in shaping the patterns of urbanization of Spain between the sixteenth and the nineteenth centuries. Professor Ringrose distinguishes different regions inside Spain with varying dynamics and characteristic urbanization patterns, revises current interpretations on the effects of the crisis of the seventeeth century and extends to the end of the Old Regime the main trends in urbanization specific to this area of soutbern europe.

KEY wORDS: Urbanization. Urban networks. Castile. Early Modern History. Markets. Institutions. Economic rationality.

\section{INTRODUCCIÓN}

Pablo Sánchez León ha desarrollado un enfoque novedoso para comprender el proceso de urbanización en Castilla durante los siglos XVI y XVI, señalando algunos puntos importantes sobre la metodología empleada tanto al estudiar este caso como el europeo en general. Con su trabajo como punto de 
partida, este ensayo pretende situar la urbanización de Castilla en un contexto más amplio, al menos en tres planos diferentes. En parte el contexto se amplía mediante la extensión cronológica del problema al siglo XVIII e incluso al XIX. También se amplía al incluir en el análisis territorios no castellanos. Y finalmente, se expande al sugerir formas alternativas de interpretar la interacción entre las ciudades.

Esta última cuestión requiere tener en cuenta que los vínculos, las redes y las jerarquias interurbanas pueden convertirse fácilmente en abstracciones que se discuten como si fuesen participantes activos en la Historia. Si bien es verdad que los trabajos de ciencias sociales están basados en este tipo de abstracciones, éstas no dejan de ser formas de explicar las acciones combinadas de individuos ${ }^{1}$. Estas redes y jerarquías urbanas representan innumerables decisiones personales, decisiones que conforman los esquemas básicos de comportamiento que acaban convirtiéndose en abstracciones históricas tales como la de «urbanización» " ${ }^{2}$ Si, como historiadores, hemos de Llegar a comprender cómo tuvo lugar el proceso de urbanización en un contexto específico, en lugar de realizar explicaciones abstractas de sus resultados posteriores, debemos considerar los objetivos y asunciones culturales de los participantes individuales.

Sánchez León ha expuesto brillantemente la lógica que subyace a nuestro modo cotidiano de analizar el proceso de urbanización europeo (y español) en la época moderna, aportando valiosos datos empiricos sobre la urbanización en Castilla y cuestionando algunos de los modos que aún se utilizan para describit y explicar este proceso. Sus cuestiones surgen en un contexto de reevaluación de las tendencias de la historia urbana en la Castilla de los siglos XVI y XVII. Su explicación incluye una crítica del análisis de la urbanización que se basa - a veces de forma poco crítica- en modelos que se apoyan en interacciones smitbianas (o derivadas del mercado). Sánchez León argumenta, por el contrario, que los factores institucionales - y presumiblemente sociales- fue-

1 Uno de los problemas presentes en la literatura teórica sobre la urbanización es que tiende a centrarse en la explicación de los orígenes de la situación actual en lugar de hacerlo en las dinámicas de los contextos históricos concretos. Teniendo en cuenta esta limitación podemos enconttar una sucinta y excelente revisión de las herramientas teóricas de la urbanización en BARNHOUSE WNLTERS, P., systems of Cities and Utban Primacy: Problems of Definition and Measurement), en TIMBERIAKE, M. (ed.), Urbanization in the World-Economy,Orlando (Fla.), Academic Press, 1985, págs. 63-86.

2 Esta idea está implicita en la observación de Jan de Vries en la que señala que el estudio de la urbanización tiene tres componentes: urbanización estructural, urbanización conductista y urbanłzación demográfica. El término surbanización conductista» incluye todo el espectro de las elecciones y los valores humanos, dificilmente evaluables. De Vries enfatiza la urbanización demográfica mientras que ignora la componente conductista. Ver de Vries, J. European Urbanization, 1500-1800, London, Methuen, 1984 [hay traducción al castellano, Batcelona, 1987]. págs. 11-12 y DE VRIES, J., «Problems in the Measurement, Description, and Analysis of Historical Urbanizationy en VAN DER WOUDE, A., HAYAMt, A. y DE VRJES, J. (eds.) Urbaniration in Histon: A process of Dynamic Interactions. Oxford, Clarendon Press, 1990, págs. 44.45.

Hippunia, LVIII/2, num. 199 (1998) 489-512 
ron importantes para el florecimiento del sistema urbano de Castilla en el siglo XVI, y quizá incluso más importantes en la desurbanización parcial de Castilla en el siglo XVII.

A su vez, defiende la singularidad del caso castellano sosteniendo de manera efectiva no sólo el hecho de que Castilla en el siglo XVI llegara a ser una de las regiones más urbanizadas de Europa, sino también que su posterior desurbanización constituye también un caso único. De este modo, sugiere, los factores sociales y la manipulación de las instituciones por parte de individuos permiten una mejor comprensión de la urbanización castellana que la que muestran las lógicas smithianas o de fuerzas de mercado.

Esta discusión se presenta como una comprobación de la validez del influyente libro de Jan de Vries, European Urbanization, 1500-1800. Sánchez León argumenta que de Vries ofrece una visión demasiado limitada de lo que constituía una ciudad. Por esta razón, el libro no dedica la suficiente atención empírica a un conjunto de centros urbanos de tamaño mediano o pequeño que funcionaban como ciudades en los niveles más bajos de la distribución de tango-tamaño que de Vries emplea para definir la urbanización. Por tamaño pequeño Sánchez León entiende las ciudades de entre cinco mil y diez mil habitantes. Además --según Sánchez León- de Vries mezcla de manera errónea las ciudades de Castilla con aquellas adscritas a la Corona de Aragón, tratándolas como pertenecientes a una única región urbana, homogeneizando de esta manera datos procedentes de dos sistemas urbanos muy diferentes. El resultado de esto sería que las observaciones de de Vries sobre España se debilitan por su confianza en los aspectos del desarrollo urbano derivados del mercado, por excluir de su análisis a las ciudades con menos de diez mil habitantes y por no tener en cuenta las diferencias regionales en el caso español, mientras que éstas sí se reconocen cuando se analizan otras partes de Europa. Aunque Sánchez León no lo señala, este resultado refuerza los estereotipos establecidos sobre la Historia de España que ahora están siendo cuestionados.

Sánchez León plantea una serie de cuestiones que operan en distintos niveles. La más inmediata es nuestra percepción del modelo de cambio de la organización urbana en Castilla en el período comprendido entre los años 1500 y 1700. Un segundo problema, no tan claramente articulado, es la evolución de la actividad urbana durante la España Moderna y hasta bien entrado el siglo XIX. Este punto refleja el problema señalado por de Vries (entre otros) al tener que decidir qué tipo de límites establecemos al comenzar el análisis de cualquier red o sistema urbano ${ }^{3}$. Las fronteras nacionales tal y como las conocemos hoy no carecen de utilidad, pero se trata de creaciones políticas modernas prácticamente carentes de relevancia para los hábitos de comportamiento interurbano hasta mediados del siglo XIX.

La tercera cuestión se refiere al concepto de urbanización en Europa en sí. Aquí no se cuestiona la aportación de de Vries cuando define una red urbana

3 IDE VRILS, J., «Measurement, Description and Analysis...», págs. 45-46, 51-52. 
de ámbito continental que emerge a mediados del siglo XVIII. Sin embargo, como señala Sánchez León, los procesos que impulsaron esa integración están aún lejos de haber quedado claros. Entre otras cosas, no hemos llegado a comprender totalmente cómo la aparición de una larga lista de ciudades capitales, sostenidas más por una distribución político-institucional de la riqueza que por actividades mercantiles, se relaciona con la integración de una red urbana continental ${ }^{4}$. En el caso castellano, por ejemplo, el resultado histórico puede no ser debido tanto a las fuerzas del mercado como lo que de Vries sostiene.

Y en un cuarto nivel -en un plano ya más general-Sánchez León nos remonta a la lógica y categorías absolutas que usamos cuando empleamos conceptos como los de lugares centrales, jerarquías de lugares centrales, distribución rango-tamaño de ciudades, y redes urbanas (u otras) ${ }^{5}$. Sánchez León nos recuerda acertadamente que los historiadores urbanos suelen ser culpables de medir lo que es fácilmente mensurable, dejando otras variables fuera de la ecuación ${ }^{6}$. Sin embargo, su presentación del caso castellano corte el riesgo de tomar partido por una falsa dicotomía entre los factores de mercado y los sociales e institucionales como explicaciones de los desarrollos observados. Esto supondría una excesiva simplificación en el análisis de las fuerzas sociales $\mathrm{e}$ institucionales.

\section{UN REPLANTEAMIENTO DEL PROCESO DE URBANIZACIÓN EN LA CASTIIIA DEL SIGLO XVI}

Independientemente de las preferencias analíticas del lector, Sánchez León nos ha hecho un tremendo servicio al separar la urbanización de Castilla de la de Aragón. Rechazando las técnicas deductivas que-de Vries utiliza para deducir la posición de las ciudades pequeñas en las distribuciones europeas de rango y tamaño, utiliza datos empíricos para incluir ciudades de entre cinco y diez mil habitantes dentro del análisis. De esta manera demuestra cómo Castilla fue una de las regiones europeas más urbanizadas en el siglo XVI, un hecho ignorado

4 Dentro de las ciudades en cuestión se incluyen Madrid, Roma, Nápoles, Viena, Berlin, Dresden, Bruselas, Lima y las capitales de varios principados alemanes de menor tamaño. Esto se discute en RINGROSE, D. R., «La cjudad y su entotno en la época moderna», Manuscrits: Revista d'Historia Moderna, vol. 15, 1997, págs. 221-245. Una versión similar en inglés, RINGROSE, D., «Capital Cities and their Hinterlands: Europe and the Colonial Dimensions, en CLARK, P. y LEPEII', B. (eds.), Capital Cities and their Hinterlands in Earby Modern Europe, Oxford, Scolar Press, 1996 , págs. $217-240$.

5 Como ejemplo, en su merecidamente famosa revision, The Making of Urban Europe, 1000-1950, Cambridge, Harvard Press University, 1985, pags. 106-136, HOHENBERG, P. y LEES, L. H., dedican un largo capítulo a "Cities in the Early Modern Economy". Ellos reconocen el auge de las ciudades políticas, pero mientras dedican partes enteras a «Urban Industry", "Economic development in Flanders and Brabant», and "The Protoindustrial City", no estudian las implicaciones económicas del auge de las capitales políticas.

6 De nuevo recordamos la posición de de Vries de concentrarse en la urbanización demográfica en lugar de en la urbanización conductista, como mencionamos en la nota 2.

Hitpania, LVIII/2, núm. 199 (1998) 489-512 
por de Vries al mezclar las referencias castellanas con las de Aragón. La nueva relación de datos presentada por Sánchez León muestra cómo Castilla despuntó también como una región que experimentó la mayor parte de su urbanización en ciudades relativamente pequeñas. También este argumento entra en discordancia con la tesis de Vries, con lo que, aceptando los planteamientos de Sánchez León debilitariamos algunas de las generalizaciones de de Vries. Este nuevo acercamiento al siglo XVI no cambia el hecho de que Castilla en el siglo XVII experimentara una grave crisis urbana. Pero si tenemos en cuenta que esta crisis afectó en mayor medida a las ciudades castellanas más grandes (excepto Madrid), la imagen de una Castilla virtualmente desurbanizada en el siglo XVII (imagen a la que yo he contribuido) tiene que ser teconsiderada.

Este desarrollo situó a Castilla al margen del contexto europeo. Aunque este sea un tema que merece nuestra consideración, el principal interés de este ensayo es la naturaleza y progresiva evolución de los sistemas urbanos en España. Sánchez León sostiene que el debilitamiento de la urbanización castellana no puede ser explicado de forma eficaz a través del mercado o las fuerzas smitbianas. El elemento principal de su análisis es una combinación de factores institucionales específicos de Castilla. Las ciudades dominantes de los niveles más altos de la jerarquía de lugares centrales castellana fueron debilitándose debido a que el poder institucional de la Corona fue desplazando las funciones urbanas de las viejas grandes ciudades hacia la emergente capital Madrid a fines del siglo XVI. Mientras, y debido a los problemas fiscales crónicos de la Corona, el Rey vendía con gran facilidad tierras baldias a compradores privados, despojando a muchas ciudades de tierras que habían sido en su momento absorbidas ilegalmente en las tierras de propios. El Rey también vendió cientos de cartas de villargo a ciudades más pequeñas '. Esto proporcionó una independencia administrativa a las ciudades más pequeñas permitiéndolas desarrollar sus propios servicios de mercado, debilitando el volumen de servicios urbanos de grandes centros regionales. Ambos factores produjeron una delegación de las funciones de lugares centrales de las grandes ciudades a las pequeñas, al ser transferidas también algunas de las actividades típicas de las grandes ciudades a la nueva capital en Madrid.

Escribiendo en la misma línea que S. R. Epstein, Sánchez León desafia tanto la ortodoxia del análisis de de Vries sobre la urbanización basada en el mercado como los estereotipos que tratan la España del siglo XVI como una entidad monolítica ${ }^{8}$. Pero del mismo modo, malinterpreta (asumiendo que se trata de una explicación monocausal ) mi propuesta de que el surgimiento de Madrid como capital residencial y política fue la principal causa del declive de

7 Entre los numerosos articulos que investigan este problema, LLOPIS AGELÁN, E., «El agro castellano en el siglo XVII. ¿Depresión o «reajuste y readaptaciones»?» en Revista de Historia Económica, vol. 4, n. ${ }^{\circ} 1,1986$, págs. 11-38, especialmente pp. 34-36.

8 Aparte de sus comentarios a este volumen, ver la introducción a EPSTEIN, S. R., An Island for itself: Economic Development and Social Change in Late Medieval Sicily Cambridge, Cambridge University Press, 1992, págs. 1-24. 
otras ciudades importantes de Castilla. Estamos de acuerdo en que la prosperidad de Madrid distorsionó y debilitó el sistema urbano de Castilla. Pero donde no estamos tan de acuerdo es en la naturaleza del sistema que estaba siendo debilitado, ya que a este respecto existen diferentes maneras de describir el proceso impulsado por el auge de Madrid".

\section{¿REDES Y SISTEMAS SIN CIUDADES?}

Mis argumentos acerca de este punto están orientadas en dos direcciones. Por un lado tengo mis dudas sobre la capacidad de explicación que pueda ofrecernos la teoria de los lugares centrales. Por otra parte, sugiero que el mundo urbano del país que hoy conocemos como España se desarrolló de manera regular y puede entenderse mejor extendiendo la aproximación de Sánchez León más allá del siglo XVI y de los límites de Castilla. Además de dividir la España peninsular en las jurisdicciones tradicionales de Castilla y Aragón debemos considerar las unidades regionales menores ${ }^{10}$. Es aquí donde podemos usar las aportaciones de Epstein relativas a la naturaleza dinámica de los mercados locales y regionales posteriores a las alteraciones causadas por la peste bubónica.

Además, dada la variedad de funciones que se acumulaban en las principales ciudades, podría ser útil conceptualizar redes de actividad como algo distinto a las redes urbanas y a la jerarquía urbana. Los vínculos dentro de estas redes resultarían de los reiterados contactos personales, familiares o comerciales entre puntos concretos. Estos contactos a menudo utilizan una o varias ciudades como puntos de enlace, ejes o centros de coordinación, pero los condicionantes interurbanos o de localización a que están sujetos son muy variables. En algunos casos, los contactos políticos, comerciales y culturales siguen pautas interurbanas paralelas. Sin embargo, la variedad de intereses entre los individuos que inician estas actividades explica que no todos los intercambios de latga distancia respondan a la misma lógica geo-económica. Esto quiere decir que

9 En este punto debo señalat que, al contrario de lo que sostiene SÁNCHEZ LEÓN, no he mantenido que Andalucía era parte de la región urbana definida por el papel de Madrid en la configuración de los movimientos de mercancías en Castilla. Por el contrario, dejo muy claro que los momentos en los que Madrid dirigió mercancias andaluzas para su suministro urbano fueron excepcionales. Ver mi libro Madrid and the Spanish Economy, 1560-1850, Berkeley, University of California Press, 1983 [hay traducción al castellano, Madrid, 1986], págs. 211-212 y 310.

10 La mayoría de la literatura teórica sobre redes y regiones urbanas destaca los factores económicos, quizás porque son los más fáciles de cuantificar. Otros trabajos prestan atención a los factores políticos, incluida la tecaudación de impuestos, pero en cambio otros tipos de interacción que están implicitos en las redes de interdependencia reciben menos atención. Ver la oportuna revisión del tema en PRAK, M., «Regions in Early Modern Europe», en Debates and controversies in economic bistory, A-sessions, Eleventh International Economic History Congress, Milán, Università Bocconi, 1994, págs. 19-56, especialmente págs. 40-43, «Economic, as compared to political and cultural regional patterns».

IHipqunia, LVII/2, nún. 199 (1998) 489-512 
las actividades básicas a partir de las cuales se construyen las redes urbanas no siempre coinciden unas con otras ".

Podemos estudiar el contexto real de los valores y motivaciones (la urbanización conductista de de Vries) que subyace a las redes urbanas al examinar no sólo las conexiones específicas en las redes, sino también las razones que motivan acciones concretas, y los patrones que éstas sugieren. Cada enlace o interacción en una red tiene un punto de origen y uno de destino; a su vez cada enlace está ligado a un lugar concreto donde alguien actúa para poner la conexión en marcha. La decisión de llevar a cabo una acción que conecte dos lugares puede darse tanto en el punto de origen como en el destino de la transacción, o en un tercer emplazamiento. Estos tres elementos de cada enlace operaban en un medio específico que incluye ciertas realidades geo-económicas ineludibles, intereses personales, y diversas trayectorias sociales de tipo familiar. Personas, información y órdenes viajan con relativa facilidad, incluso por vía terrestre. Hasta cierto punto esto también puede decirse de un buen número de bienes de lujo de gran valor, destinados a consumidores dispuestos a pagar costes de transporte muy elevados. Las mercancías pesadas, voluminosas o de poco valor son mucho menos dadas a recorrer largas distancias, especialmente por tierra, ya que el coste del transporte las encarecía demasiado para la mayoría de los consumidores que se encontraban lejos del punto de origen ${ }^{12}$. Estos diferentes condicionantes en el traslado de personas e información en oposición al transporte comercial son extremadamente importantes en un mundo preindustrial y premoderno. El primer tipo de actividad puede desarrollar conexiones sotprendentemente densas en las largas distancias, mientras que el segundo tipo, normalmente transporte de alimentos, combustible, materiales de construcción y manufacturas de uso cotidiano, se define mediante, y ayuda a crear, sistemas de lugares centrales locales y regionales ${ }^{13}$.

Las implicaciones de esta situación para una región como Castilla entre el 1500 y la llegada del ferrocarril incitan a una aproximación dual a nuestras explicaciones sobre la urbanización, una dualidad basada en la aparente diferencia entre mercados e instituciones como factores que configuran la urbanización. De hecho, la diferencia entre la facilidad de transporte/comunicación de los individuos y la información comparada con las limitaciones de movilidad de mercancías pesadas ayuda a explicat por qué Madrid pudo gobernar un impe-

11 Estas ideas son inherentes a los modejos de lugares centrales, Jo cual acentúa la relativa facilidad con la cual las difexentes actividades pueden ser realizadas a distancia. El desar rollo del marco teórico original, con una bibliografia básica, está recopilado en SMITH, C. A., «Types of City-Size Distributions: A Comparative Analysis», en VAN DIR WOUDE, HAYAMI y DE VRIES, Urbanization in History..., págs. 20-22.

12 Esta observación no es nueva, y fue expuesta de una manera interesante hace algunos años por el geográfo económico FOX, E. W., en su History in Geographic Perspective, New York, Norton, 1971.

${ }_{13}$ Una manera de enmarcar esta distinción en términos teóricos es ofrecida por HOl ILNBERG y L.EES, The Making of Urban Europe..., págs. 4-7. 
rio mundial y a la vez soportar hambrunas cuando las malas cosechas reducian los suministros de alimentos de un 15 a un 20 por ciento en un radio de 200 kilómetros de la ciudad ${ }^{14}$. Si nos fijamos demasiado detenidamente en el último problema, como yo he hecho alguna vez, es fácil observat las redes y jerarquías urbanas en términos de ciertos tipos de intercambios comerciales. $\mathrm{Si}$ ponemos énfasis en la naturaleza aristocrática y propietaria de las elites castellanas, y en las líneas de autoridad política que emanaban de la Corona y del Consejo de Castilla contemplamos, sin embargo, un sistema urbano muy diferente. Ese tipo de sistema está también centrado en Madrid, pero refleja la subordinación de las elites rurales y urbanas a los asuntos de Madrid en una mayor escala geográfica. En este caso la relación entre individuos participantes y Madrid es de naturaleza social, administrativa y fiscal, en vez de económica. Este tipo de unión necesita menos de los lugares centrales intermedios.

Esta ted, amplia geográficamente hablando, sólo tiene sentido cuando es analizada en términos socio-políticos. Más allá de la región geográfica que proveía a la ciudad con suministros básicos, encontramos regiones que, aunque unidas políticamente a Madrid, deben también ser definidas por patrones autónomos y coherentes de intercambio económico que producian jeratquías de lugares centrales basadas en centros regionales con apenas interacción económica con Madrid. Este punto requiere dos observaciones adicionales. (1) El lugar central en este sistema regional coordinado derivado del mercado no tiene por qué ser un centro político de importancia, y puede incluso parecernos pequeño en términos de población ${ }^{15}$. (2) Los límites entre regiones económicas, inmersas o no dentro de un sistema político más grande, son inevitablemente vagos e inestables.

Cambios en la geografía de la demanda o en la técnica del transporte hacen que esos límites varien. Diferencias sutiles en los análisis coste-beneficio de los proveedores individuales de bienes, y el variable valor intrínseco de las mercancías dentro de la categoría "suministros básicos» implican que algunas áreas rurales enviarán mercancías a más de una red regional, dificultando así el trazado de límites claros entre las regiones que se definen por su comportamiento comercial. Esto se aprecia particularmente en una región como Castilla, que dependía enteramente del transporte por vía terrestre en forma de carros de bueyes o bestias de carga. La extensión y la dirección de los vínculos económicos en ese entorno variaba enormemente y dependía de muchos factores además del precio y la demanda en los destinos potenciales.

Como ejemplo, las decisiones sobre transacciones individuales se veían afectadas por la demanda temporal de mano de obra en la agricultura y por la posibilidad de traer una carga de mercancías en el viaje de vuelta. Normal-

14 Las distancias a las cuales acudian las autoridades urbanas para asegurar un suministro estable de grano en una región de abastecimiento inestable son sugeridas en RINGROSE, D., Madrid and the Spanisb Economy... págs. 145-154 y 203-205.

15 Uno piensa aquí en Bilbao, que fue un centro importante de comercio y de banca, con sólo 11.000 habitantes en 1800 .

Hitpania, LVIII/2, núm. 199 (1998) 489-512 
mente pensamos en estos vínculos económicos como movimientos unidireccionales de mercancías desde su origen hasta el mercado, pero el campesino/transportista rural lo veía de un modo más complejo. Si el transportista podía esperar realizar un cargamento de vuelta tras haber distribuido los bienes que transportaba, sus cálculos con respecto al beneficio obtenido y a las tasas de transporte eran bien diferentes de aquéllos hechos sobre la base de una única entrega de mercancías. Si el transportista empleaba animales de tiro cuando éstos no eran necesarios en la cosecha y esos animales habían de ser alimentados tanto si trabajaban como si no, le merecía la pena el esfuerzo de emplearse en el transporte incluso si las ganancias sólamente cubrían una parte del coste de mantenimiento de sus animales. Esto explica que, en determinadas estaciones del año, el transporte hacia el mercado fuese más barato que en otras.

Además de las muchas formas de transacciones interurbanas que reflejan de por sí la necesidad de comunicación, de dar órdenes, o de poner mercancías en el mercado, existe un tercer factor que contribuye a nuestra descripción de las redes urbanas. Se trata del movimiento de población y mano de obra hacia y desde las ciudades. Sólo unas pocas ciudades preindustriales eran demográficamente autosuficientes. Cuanto más grande era una ciudad, peores eran las condiciones higiénicas y mayor era la necesidad de un flujo constante de inmigrantes rurales para sostener y expandir su población y su mano de obra. Al contrario de lo que los historiadores sostenían hace treinta años, hoy sabemos que en el campo de los siglos XVI y XVI la gente se movía con extraordinaria libertad ${ }^{16}$. En una sociedad basada en el trabajo intensivo y con una débil tecnología, el trabajo es el factor decisivo de producción en cualquier actividad productiva. Así, la migración laboral es comparable al comercio de bienes básicos y suministros, ya que en ambos la demanda es abundante e inelástica. Pero a diferencia de las mercancias pesadas, los individuos se mueven con mayor facilidad y así, leñadores, campesinos y mendigos urbanos viajaban habitualmente cientos de kilómetros a través de España. De hecho, se movían en un marco geográfico parecido al de las transacciones administrativas y de integración de elites de más larga distancia. La demografía de las ciudades, sin embargo, muestra cómo la inmigración urbana se producía normalmente entre el campo y la ciudad, y sólo en raras ocasiones se producían migraciones de una ciudad a otra. Esto significa que si consideramos la inmigración como una actividad económica, las regiones que exportaban grandes cantidades de mano de obra a una ciudad como Madrid, apatecen como parte de un gran sistema urbano orientado hacia Madrid. Asturias, por ejemplo, suministró el mayor contingente unitario de inmigrantes a Madrid durante la mayor parte del siglo XIX. Pero aparte de esta inmigración, Asturias tenía poca interacción económica con la capital. En la misma línea, la Andalucía de la época moderna envió

16 VAsSBERG, D., The Village and the Outside World in Golden. Age Castile: Mobility and Migration in everyday Rural Ljfe, Cambridge, Cambridge University Press, 1996. 
relativamente poca gente a Madrid, aunque los vínculos fiscales y políticos eran muy estrechos ${ }^{17}$.

Lo anterior sugiere que si pretendemos comprender el significado de la urbanización dentro de las dinámicas sociales y económicas en un país preindustrial como España, debemos separar la palabra «urbano» de la palabra "red» y observar las características de redes funcionalmente distintas. Por ello, antes he mencionado algunas de esas redes: autoridad jurisdiccional (y los que la implementan), el mundo social de la elite propietaria, el mundo comercial de bienes de hijo, el mundo de mercancías agrarias de bajo coste, y las redes definidas por los movimientos de población y trabajo.

Si pretendemos apuntar hacia los orígenes de nuestro mundo urbano, es lógico hacer énfasis en aquellas redes asociadas a las mercancías pesadas y a la autoridad política centralizada, ya que de una manera relativamente directa presagian el surgimiento de la economía de metcado y del estado. Pero si lo que queremos es entender las dinámicas de la urbanización de la época moderna en su propio contexto, debemos fijarnos en factores que en la sociedad de ahota pueden parecernos secundarios, como por ejemplo los imperativos del estatus familiar. Pero en ambos casos, es esencial tener en cuenta todas las dimensiones del problema sin poner demasiado énfasis en una u otra explicación. Dichas estas observaciones, veamos qué podemos aportar a la discusión del proceso de urbanización en España.

\section{DE REGRESO A LA CASTILLA DEL SIGLO XVI}

Para comenzar debemos tener claro cuál es el objeto de nuestra discusión, es decir, qué es lo que en realidad ocurría en el siglo XVI cuando la población urbana de Castilla crecía más rápidamente que la de la región. Sánchez León argumenta que, en contra de las asunciones incluidas en mis comentarios previos sobre esta situación, la red urbana que emergió en Castilla en el siglo XVI no fue el resultado de un sistema de mercado integrado con una jerarquía de centros de servicios que tenía su cumbre en Toledo. Sánchez León ofrece una importante alternativa al sugerir que la urbanización castellana fue desde su inicio similar al Madrid de los siglos XVII y XVIII. Esta ciudad era, fundamentalmente, un centro residencial para las elites aristocráticas terratenientes y burocráticas que ocupaban y controlaban las instituciones centrales de las monarquías de los Borbón y de los Habsburgo. Como ya he mostrado, la base de la economía urbana la constituían, sobre todo, los impuestos y recursos fiscales de la Corona y los ingresos basados en la renta de las elites terratenientes y del cleto ${ }^{18}$.

17 Debo el impulso de destacar la relación de la inmigración urbana al concepto general de los sistemas urbanos a los acertados comentarios de DAVID-SVEN REHIER. Ver su reseña a mi libro más reciente, «Milagro español: Si pero menos», Reyista de Libros, núm. 4 , Madrid, 1997, págs. 21-24.

18 RINGROSE, Madrid and the Spanish Economy..., págs. 71-81.

Hipania, LVIII/2, תúm. 199 (1998) 489-512 
Desde el punto de vista de Sánchez León, las otras ciudades que dominaban Castilla antes del surgimiento de Madrid fueron versiones en tamaño más pequeño de esta misma ciudad. Desde esta perspectiva, la contribución de $\mathrm{Ma}$ drid a la urbanización de Castilla no supuso el desmantelamiento de una jerarquía de lugares centrales, definida sobre la base del mercado, sino simplemente la consolidación de las elites rentistas y terratenientes preexistentes dentro de una única ciudad. Sánchez León refuerza este argumento de manera efectiva haciendo uso de datos empíricos sobre ciudades de menos de diez mil habitantes para invalidar conclusiones deducidas del patrón jerárquico de las ciudades más grandes. Una vez que estas ciudades de tamaño menor son incluidas, el patrón deja de ser el de un sistema urbano dirigido por el mercado. Lo que podemos extraer de estas conclusiones es la evidencia de que, mientras Castilla tenía un grado de urbanización alto para el modelo de la época, una gran parte de la población urbana se concentraba en ciudades de menos de diez mil habitantes. Estas ciudades, por definición más próximas a la sociedad rural y menos diferenciadas económicamente, aparecen como candidatas pobres a una integración seria con los intercambios comerciales a larga distancia.

Estas conclusiones ofrecen un correctivo saludable a las observaciones basadas en la inferencia de historiadores urbanos como de Vries o yo mismo. En este punto me gustaría sugerir un espacio neutral para la discusión en el que se aprovechasen tanto los argumentos smitbianos como los institucionales. Si bien los resultados pueden no proporcionar respuestas definitivas, quizá ayudarán en la formulación de nuevas cuestiones que reconduzcan la investigación. En mi propio trabajo presenté, tiempo atrás, una imagen de la urbanización castellana en el siglo XvI como un proceso que envolvia una bastante "estandap» jerarquía de centros urbanos por tango-tamaño incluidos en el área de las dos mesetas (o lo que es lo mismo, Castilla la Vieja/León, Castilla la Nueva/Extremadura/La Mancha) ${ }^{19}$. Describí éste como un sistema de ciudades basado en, o al menos que reflejaba, una jeratquía de centros urbanos anclados en Toledo y ligados entre sí por una red de relaciones comerciales.

Sánchez León sugiere que la interdependencia de mercado aquí implícita era, de hecho, deducida de la presencia de una jeratquía de rango-tamaño de ciudades similar a las jerarquías asociadas a mercados integrados y que no estaba basada en datos empíricos. Es cierto que existía poca información sistemática sobre los tamaños de las ciudades cuando escribí mis comentarios, pero este punto puede haberse, de algún modo, exagerado. Existe cierto número de estudios que documentan actividad industrial y comercial de larga distancia en el interior de Castilla. Varias ciudades castellanas - Toledo, Segovia, Ávila, $\mathrm{Pa}$ lencia, Cuenca, Béjar- albergaton industrias textiles laneras. En muchos casos sus textiles eran vendidos lejos de las regiones en las que se producían, llegando

19 Si mantuve alguna vez que Sevilla estaba integrada en esta red regional, no lo haría ahora. Había importantes conexiones entre Sevilla y Medina del Campo y Toledo, pero de signo diferente a aquéllas existentes dentro de la región. 
en alounos casos a los mercados internacionales ${ }^{20}$. Existía además, una grande y compleja industria que producía y exportaba lana en bruto. Esta requería no sólo de pastos y cañadas, sino también de lavaderos, centros de embarque regionales y una coordinación de las exportaciones a través de Bilbao, en el norte, o a través de los puertos mediterráneos y Sevilla. El gran comercio y las ferias de Medina del Campo, Medina de Rioseco y Villalón formaban parte de una red financiera y de comercio que también incluía las lanas y textiles castellanos. La comunidad mercantil de Toledo proporcionaba bienes al por mayor y funciones de gestión comercial pata la distribución al por menor de especias importadas y bienes de lujo a numerosos lugates en el initerior de Castilla. Otra investigación nos habla de una constelación de ferias locales y regionales donde textiles de producción regional entraban en el sistema de redistribución y donde bienes importados eran vendidos en los mercados locales por comerciantes toledanos. Toledo en sí no era sólo un centro de producción de lana, sino que además producía o coordinaba la producción de seda, lino y armas ${ }^{21}$.

Una imagen global de la economía castellana nos muestra que la mayor patte de la misma reposaba sobre la agricultura y los beneficios agrarios, asi como que sus rentas eran obviamente la mayor fuente de riqueza para las elites. Así, los hábitos residenciales de las elites estaban destinados a entrar en relación con la localización de los servicios urbanos. No hay duda de que esta lógica, junto con la expansión de la población rural y de los cultivos, explica el incremento del número de habitantes que poblaban las ciudades de tamaño pequeño y mediano y que estaban asociadas a pequeñas comarcas económicas.

Sin embargo, la presencia y el crecimiento de ciudades mayores requiere una explicación más compleja. Algo tiene que mover a las elites profesionales y terratenientes de fuera de la región inmediata para que la ciudad llegue a acumular una gran población. Históricamente, en Castilla los factores que contribuyeron con claridad a este proceso fueron el establecimiento en las ciudades de las casas aristocráticas verdaderamente pudientes, así como de las sedes episcopales y arzobispales más grandes y de las agencias regias importantes. Estos factores explican en gran medida la relativa importancia de Valladolid y Toledo dentro de Castilla la Vieja y Castilla la Nueva. Valladolid era la sede de un rico arzobispado y de la Chancillería de una gran parte de Castilla. Toledo, por su parte, era la tesidencia del arzobispo y primado de la Iglesia española, así como la residencia favorita de Carlos $V$ cuando éste se encontraba en el país.

En este contexto, la actividad industrial y comercial descrita previamente se ajusta a los patrones urbanos inspirados por estructuras sociales e institucionales y realiza sus propias contribuciones al sistema de red. Ciudades como Cuen-

20 Ángel García Sanz señala que las 40 ciudades más grandes de Castilla crecieron un 75 por ciento, mientras que la población general creció solamente un 40 por ciento entre $1530 \mathrm{y}$ 1590. También apunta que en torno al año 1585 , el volumen y calidad del tejido de lana producido sólo en Segovia, eta comparable al producido en Florencia en las mismas fechas. GARCía SANZ, A. «Auge y decadencia en España en los siglos XVI y XVII: Economía y sociedad en Castilla", Revista de Historia Económica, vol. $3, \mathrm{n},{ }^{0} 1,1985$, págs. 14 y 17-19.

21 RINGROSE, Madrid and the Spanish Economy..., págs. 253-254 y 278-294.

Hitpasia, LVIIT/2, núm. 199 (1998) 489-512 
ca, Ávila, Béjar, Talavera y Segovia habrín sido mucho menos prominentes en el siglo XVI sin sus componentes industriales. Medina del Campo, sin las grandes fetias cometciales, pasó rápidamente a ser insignificante. Toledo, por el contrario, entró precipitadamente en declive aún manteniéndose como residencia del arzobispo y primado de la Iglesia española. Mientras cabe la duda al especificar las conexiones causales exactas, existe una correlación estrecha entre el declive de un número indeterminado de ciudades grandes y el de sus bases comerciales.

Hasta cierto punto la discusión recuerda el argumento proverbial de si fue primero la gallina o el huevo. La esencia de un sistema urbano en una civilización agraria es, inevitablemente, la agricultura y la sociedad basada en esa agricultura. Pero tanto los factores políticos como comerciales trascienden a menudo la importancia local de una agricultura preindustrial, estimulando la aparición de más y más amplias redes de interacción. Allí donde estas redes encuentran o se acomodan a ciudades ya establecidas $-\mathrm{y}$ por tanto a mercados ya existentes - las ciudades acogen un gran abanico de funciones. Allí donde se imponen las circunstancias de ubicación y cantidad de recursos, las ciudades tendrán componentes de tipo industrial y comercial relativamente importantes, independientemente de los factores políticos o de elites residentes.

Esto sugiere ciertas deducciones generales sobre la urbanización castellana en el siglo XVI. Sánchez León ha mostrado de manera convincente que el incremento de la expansión urbana en la franja inferior del patrón rango-tamaño no sólo documenta una Castilla altamente urbanizada, sino también una Castilla en la que la urbanización se sustentaba en el crecimiento de pequeñas "ciudades capitales" caracterizadas por economías agrícolas, basadas en rentas y dominadas por oligarquías de naturaleza terrateniente. Esto constituye una aparente excepción o inexactitud en el análisis de de Vties.

Este es un importante descubrimiento y coincide con la percepción que tenemos del campo castellano. Al mismo tiempo, sin el rango de funciones urbanas generadas por una vida económica relacionada con el mercado, el modelo general de urbanización castellana se habría parecido aún menos al modelo rango-tamaño que de Vries atribuye a Europa en general. Algunas ciudades grandes y muy grandes - Segovia, Valladolid, Toledo - habrían sobresalido en cualquier caso como consecuencia de las elites administrativas y rentistas que hospedaban. Pero es improbable que hubieran adquirido el tamaño que alcanzaron de no haber recibido el apoyo de y de no haber contribuido a abastecer y coordinar una red mercantil regional suficientemente integrada.

\section{EL «DEBILITAMIENTO " URBANO Y LA *CRISIS" DEI, SIGLO XVII}

Lo que esto quiere decir, y aquí nos acercamos de nuevo a las conclusiones de Sánchez León, es que debemos ser cuidadosos en la manera de presentar explicaciones sobre el declive de la urbanización en Castilla que marcó el siglo XVII. Considerar los factores institucionales o los de mercado como principales explicaciones del debilitamiento sería como escuchar el sonido de una sola mano cuando se está aplaudiendo. Pero también es importante advertir que los 
argumentos como los de Sánchez León o los de Epstein facilitan la comprensión de lo que estaba ocurriendo en el mundo urbano castellano de los siglos XVII y XVIII. La idea predominante es aquélla en la cual Madrid se convirtió en el centro macrocéfalo de una red de ciudades debilitadas. En este proceso, todos los centros importantes del siglo XVI ven rebajadas sus poblaciones a niveles de entre diez mil y veinte mil habitantes, con la excepción de Valladolid, que se mantiene con una población sensiblemente mayor ${ }^{22}$.

El crecimiento de Madrid y el declive de las otras grandes ciudades castellanas no se produce por el simple resultado de la reconstrucción forzada de una red mercantil castellana impulsada por la autoridad política. La coerción regia en efecto era parte del proceso, pero el desenlace es resultado de al menos tres tendencias interrelacionadas. Una de ellas es el surgimiento de una administración real con una considerable capacidad de crear impuestos y de ofertar juros. Esto, unido a la atracción de mandos militares, de puestos en los consejos reales, embajadas, y del prestigio de la proximidad al Rey y su hacienda, motivó que una gran parte de las elites propietarias empezara a construirse grandes residencias en Madrid, y con ello, a gastar gran parte de sus haciendas allí. Pero vivir en la Corte suponía grandes gastos, con lo que era más probable atraer inmigrantes nobles de las grandes ciudades residenciales del siglo XVI. El consiguiente crecimiento de Madrid motivó la intervención real en los mercados tegionales en busca de alimentos y combustible para garantizar el adecuado aprovisionamiento de la Corte y la ciudad. Estas intervenciones se acentuaban en épocas de cosechas escasas. Esto creó una inevitable presión al alza en los precios que otras ciudades en el mismo sistema de distribución castellana se vieron forzadas a pagar por víveres y combustibles.

En un contexto paralelo, la combinación del crecimiento de población con el descenso del rendimiento per cápita en la agricultura también creó inestabilidad en el abastecimiento urbano en años en los que las cosechas eran pobres. En el curso de las cuatro o cinco décadas posteriores a 1580, los precios de los alimentos en Castilla empezaron a crecer más rápidamente que los precios de otras mercancías menos básicas. Y teniendo en cuenta que la economía castellana incluía un sector industrial, éste era doblemente vulnerable al aumento de los precios alimentarios, y consecuentemente al de los salarios básicos, mientras que los precios de los productos industriales crecían más lentamente. En este entorno vemos cómo las competitivas industrias de los Países Bajos no

22 Existe un desacuerdo sobre el tamaño real de Madrid y sobre la cronología de su más rápido crecimiento, pero hay un consenso general sobre la contracción de las principales ciudades castellanas. Pocos historiadores del siglo XVII de España han aceptado mi estimación de que Madrid podía haber tenido al menos 175.000 habitantes en 1630. Maria Carbajo Isla estima un total aproximado de 130.000 , y ha demostrado convincentemente que Madrid tenía al menos 90.000 habitantes en una fecha tan temprana como 1600 , comparado con mis anteriores estimaciones de 65.000. RINGROSE, Madrid and the Spanish Economy..., págs. 43-44 y Apéndice A, págs. 331-333; CARBAJO ISI.A, M., La población de la villa de Madrid desde finales del siglo XVI basta mediados del siglo XIX, Madrid, Siglo XXI, 1986.

Hispunia, LVIII/2, núm 199 (1998) 489-512 
sólo disfrutaban de salarios y precios más estables, sino que además aprendieron a producir textiles menos elaborados que necesitaban menos trabajo. Esto debilitó los productos manufacturados españoles tanto en los mercados interiores como en los extranjeros cuando la tendencia de los precios locales incrementó los costes de trabajo. Por eso no es tan sorprendente que el comercio de textiles de alta calidad en las grandes ciudades descendiera bruscamente a principios del siglo XVII.

De hecho, dos factores estaban debilitando las grandes ciudades del interior al desmantelar o reconducir dos de las más importantes redes de actividad que dan sustancia a una red urbana. En el plano institucional, los castellanos ricos se estaban trasladando a Madrid y gastando su dinero allí. Y en un plano smithiano, observamos cómo las tendencias económicas interiores y extranjeras debilitaron las partes del sistema urbano derivadas de la actividad mercantil. Esto no es nuevo, aunque deben apuntarse una o dos modificaciones. El proceso no es ni institucional ni smitbiano, sino que implica una interacción de los dos. Además, el proceso es más fácil de entender usando la idea de diversas redes de interacción que cubren la misma geografía, pero que responden a diferentes condicionantes y motivaciones personales.

Esto es más fácil de sostener si recordamos el énfasis anteriormente puesto en el desarrollo urbano de las numerosas ciudades de menos de diez mil habitantes. Aquí coincido con Sánchez León en diversos puntos. Estas ciudades exan menos vulnetables a la marcha de los rentistas a Madrid, a la distorsión en los mercados de mercancías por medio de la coerción desde Madrid, y al declive de las manufacturas. Sin embargo eran vulnerables a la subida de las rentas de la tierra y de los impuestos. Cuando los precios de los alimentos subían, los terratenientes en toda Europa transformaban las tierras de pasto en tierras de labranza beneficiándose de la sobreabundancia de potenciales arrendatarios para subir las rentas y recortar los salarios rurales. Mientras, la monarquía subía desesperadamente los impuestos y rentas públicas. Esto llevó a la venta de tierras baldias y villazgos antes mencionada, a la colaboración entre las elites locales y la Corona, y a la usurpación de las comunales tierras de propios por parte de las oligarquías locales ${ }^{23}$. El incremento contínuo de impuestos regios a las ciudades incitaron a las oligarquías gobernantes locales a vender censos municipales para responder a las demandas de la monarquía. Esas mismas oligarquías, como individuos privados, compraban después esos mismos censos. Y para pagar el coste de los réditos anuales creados, solicitaban permiso para subir los impuestos locales. Esto implicaba no sólo una más rigurosa recaudación de la alcabala, sino también de los impuestos indirectos en el vino, aceite de oliva, carne, y papel, así como de los portazgos, corredurías y muchos otros impuestos menores, la mayoría de los cuales no afectaban a la nobleza ni a las oligarquías que controlaban el gobierno de la ciudad. De esta compleja manera, la subida de impuestos y los préstamos de la Corona eran asumidos por una so-

23 Ver, por ejemplo, LIOPIS AGEI AN, (El agro castellano en el siglo XVII...», págs. 34-36. 
ciedad rural ya enfrentada por las cosechas cada vez más escasas y variables ${ }^{24}$. Un efecto de esto fue la pérdida de tamaño de muchas pequeñas ciudades; otra fue la redistribución del control de la tierra en favor de las oligarquías locales y el aumento de la disparidad en la distribución de la renta.

Esta interacción entre la capacidad impositiva y la dependencia de las oligarquías municipales respecto de la Corona es la tercera y más generalizada fuerza que redefine el sistema urbano, y se puede considerar claramente institucional en el sentido en el que Sánchez León utiliza el término. Mientras que los otros dos factores señalados anteriormente afectan más directamente a las grandes ciudades, transfiriendo servicios y consumo urbanos a Madrid, el impacto del factor institucional es mayor y afecta más profundamente en las pequeñas ciudades agratias. La diferencia está, sin embargo, en que este tercer proceso no destruyó estas pequeñas ciudades, sino que transformó su estructura interna ${ }^{25}$. Esta observación no supone una novedad, pero aporta una sutil difetencia en la manera de analizar la "desurbanización» de Castilla en el siglo XVII. De este modo, al incluir estas pequeñas ciudades en la discusión podemos introducir el tema de la «desurbanización» en el más amplio debate sobre si la economía en sí experimentó un declive o sufrió una reorganización ${ }^{26}$. Esta última aproximación es la que ha venido a dominar la cuestión, haciendo más fácil hablar de un sistema urbano que sufrió una descentralización en lugar de un virtual desmantelamiento ${ }^{27}$. También proporciona un terreno de análisis común con la discusión de Epstein sobre la Sicilia tardomedieval, así como una

24 Esta creciente interdependencia entre las oligarquías locales y la Corona es vista en los aspectos financieros de la venta de fueros de villazgo discutidos por NADER, H., Liberty in $A b$. solutist Spain: The Habsburg Sale of Towns, 1516-1700, Baltimore, John Hopkins University Press, 1990. Un proceso paralelo tuvo lugar en Francia. Ver, por ejemplo, BEIK, W., Absolutism and Society in Seventeenth-Century France: State Power and Provincial Aristocracy in Languedoc, Cambridge, Cambridge University Press, 1987.

25 A modo de ilustración, entre 1586 y 1690 Ciudad Real sufrió una caída en el número de trabajadores en el sector primatio del $15 \%$, un $48 \%$ en el secundario y un $53 \%$ en el sector terciario. Entre 1590-95 y 1752 Ocaña experimentó una caída del $21 \%$ en el sector primario, un $58 \%$ en el secundario y un incremento del $23 \%$ en el sector terciatio. LÓPEZ-SALAZAR PéREZ, J., Estrucuturas agratias y sociedad rural en La Mancha (ss. XVI-XVII), Ciudad Real, Instituto de Estudios Manchegos, 1986, págs. 21-27.

26 Esto, por supuesto, se refiere al clásico artículo de LLOPIS AGELAN, «El agro castellano en el siglo XVII..., págs. 11-27, y el debate relacionado presentado en THOMPSON, I. A. A. y Yun Casalilla, B. (eds.), The Castilian Crisis of the Sevententh Century: New Perspectives on the Economic and Social History of Seventeenth-rentury Spain, Cambridge/Madrid, Cambridge University Press/Instituto de Estudios Fiscales, 1994.

27 Aquí La Mancha proporciona un interesante ejemplo que refuerza la argumentación de Sánchez León. Entre 1591 y 1693, cuando La Mancha perdió el 13\% de su población, de sus 35 ciudades de entre 2.500 y 7.500 habitantes, sólo 13 perdieron más del $15 \%$ de su población. De las 22 que conservaron al menos un $85 \%$ de su población, 12 eran de hecho más grandes en 1693, y cuatro de éstas habian crecido más del $15 \%$. Vemos claramente que la red de modestas ciudades agrarias que analiza Sánchez León resistió la crisis del siglo XVII con facilidad. LóPEZ-SALAZAR, Estructuras agrarias..., págs. 59-61 y 671-675.

Hispania, LVIII/2, núm. 199 (1998) 489-512 
manera de interpretar la recuperación castellana que comienza a finales del siglo XVII y se extiende a través de la mayor parte del siglo XVIII ${ }^{28}$.

\section{CASTILLA EN EL SIGLO XVIII}

¿Qué hay de la urbanización española del siglo XVIIr? Aquí merece la pena tratar el proceso en dos niveles geogtáficos y retornat a las ideas articuladas previamente sobre las «redes de actividad» como algo distinto de las descripciones corrientas de sistemas urbanos. Esto puede también resolverse de maneta sucinta puesto que la mayoría de mis proposiciones al respecto han sido expuestas recientemente ${ }^{29}$. Al menos conceptualmente, he propuesto un sistema urbano regional interior o castellano que se correspondería con la mayoría del territorio de Castilla desde la Cordillera Cantábrica hasta Sierra Morena. Aunque no muy centralizado, era éste un sistema de ciudades coordinado de manera central por dos realidades. La actividad económica que la mayoría de la región compartía, la cual no sobrepasaba los límites regionales, era la participación en varios niveles para abastecer a Madrid de alimentos, combustible y bienes manufacturados de bajo coste. La segunda característica que tenían en común era política y administrativa. Todas las grandes ciudades estaban ditectamente ligadas a Madrid por medio de tribunales regios, corregidores e intendentes. Es conocido que esta conexión política también se extendía a otras regiones de la España peninsular, pero sólo en el interior de Castilla la red política era tan estrechamente paralela a una actividad económica que operaba cada vez más como un sistema de mercado.

Es importante señalar que en muchos aspectos era una red urbana por defecto, por falta de integración en cualquier otra. Ésta era la herencia de la descentralización de la vida urbana en la Castilla del siglo XVII, que dejó muy pocas partes de Castilla con lazos económicos persistentes fuera de su interior. Al mismo tiempo pueden señalarse una variedad de intercambios económicos dentro de Castilla que reflejarian especializaciones tegionales del tipo sugerido por Epstein. Las especializaciones regionales en trigo, cebada, maderas, catbón, vacuno, bovino, porcino, lana transhumante, lana local, vino, aceite de oliva, etc. sustentaban los vínculos entre comarcas que a menudo estaban separadas por distancias considerables. Algunas de aquellas transacciones alcanzaban a su

28 La suerte de dicha «recuperación» a finales del siglo XVII es también un tema que necesita revisión. No hay ninguna duda de que algunas tensiones malthusianas iban apareciendo en Castilla en la década de 1770 , pero el proceso global de reajuste peninsular y de adaptación a las condiciones de mercado de la Europa Atlántica del siglo XVIII permite dejar abierto al debate la naturaleza de esa inminente crisis. Ver mi libro Spain, Europe, and the «Spanish Miracle», [hay traducción al castellano, Madrid, 1996], págs. 55-80.

29 Salvo observaciones al contratio, los siguientes comentarios sobre redes urbanas regionales en España están sacados de mi Spain, Europe, and the «Spanish Miracle», capitulos 8-11. También se encuentra en los mismos capítulos de la edición española, España, 1700-1900: El mito del fracaso, Madrid, Alianza, 1996. 
vez varios mercados costeros, pero excepto el comercio de lana vía Bilbao y las exportaciones de trigo vía Alicante, son más notables por su variedad que por su volumen ${ }^{30}$.

Es ésta una descripción de Castilla como una red de mercados regionales y de ciudades que encajan muy bien con el tipo de urbanización que Sánchez León nos ha mostrado. Es también un modelo de dinamismo económico que, de alguna manera, entra en conflicto con sus generalizaciones sobre el estancamiento económico causado por la concentración de propiedad en manos de las pequeñas elites locales cuyos sistemas de valores etan opuestos a los del empresario capitalista. En la práctica, estas generalizaciones se ven invalidadas cuando se analiza con más detalle un área tan vasta como Castilla.

En el transcurso de los siglos XVIII y XIX encontramos en Castilla varios ejemplos locales tanto de dinamismo como de estancamiento, en la medida en que las diversas oportunidades económicas interactuaban con circunstancias locales específicas. Un ejemplo es la reorientación de los productores castellanos de trigo hacia el mercado atlántico de finales del siglo XVIII en adelante ${ }^{31}$. En la aparentemente aislada Sierra de Gredos los campesinos reorientaron sus actividades de forma drástica entre 1750 y 1850 , en un área en la que el transporte era un problema crónico ${ }^{32}$. Agustín González Enciso nos ha aportado una fascinante introducción a una gran variedad de actividades protoindustriales que emergieton en la Castilla del siglo XVIII. Gran parte de esta actividad estaba destinada a fracasar ya que las condiciones de mercado cambiaron drásticamente en el siglo XIX, pero es un error otorgar a este proceso la etiqueta de "declive "o "estancamiento" sin tratar de comprender que éste formaba parte de un proceso de adaptación más amplio ${ }^{33}$. En línea con una versión adaptada al siglo XVIII del estudio empírico de Sánchez León sobre el siglo XVI, yo mismo realicé una vez una comparación de los cambios de tamaños de los pueblos en las provincias de Ávila y Toledo, entre 1787 y 1887. Las comarcas que se habían llenado de pueblos dinámicos en el siglo XVI a menudo permanecieron como reductos agrarios en el último periodo en ambas provincias. Otras áreas, tales como el sur de la provincia de Toledo, vieron cómo comunidades antes minúsculas llegaban a ser centros textiles de tamaño considerable que servían a los mercados regionales y a los sectores menos acomodados de

30 Aunque sus conclusiones pueden ser consideradas ahora incompletas, algunos de estos intercambios regionales están documentados en RINGROSE, D., Transportation and Economic Stagnation in Spain, 1750-1850, Durham, Duke University Press, 1970. Un detallado apéndice de la edición española testimonia este tráfico, Los transportes y el estancamiento económico de España, 1750-1850, Madrid, Tecnos, 1972.

31 YUN CASALILLA, «Mercado de cereal y burguesía...», págs. 71-72.

32 HILES-BURKHOLDER, S., «From Wool to Wine: Demographic and Economic Change in the Sierra de Gredos, 1750-1887 (Ph.D. Dissertation, University of California, San Diego, 1990).

33 Ver GONZÁLEZ ENCISO, A., «La proto-industrialización en España» y «La proto-industrialización en Castilla la Vieja en el siglo Xvirl», en Revista de Historia Económica, vol. $2, \mathrm{n} .{ }^{\circ} 1$ y n. ${ }^{\circ} 3,1984$, págs. 11-46 y 51-82, respectivamente.

Hipania, LVIII/2, núm. 199 (1998) 489-512 
la sociedad de Madrid ${ }^{34}$. También merece la pena tener en cuenta cómo la nobleza terrateniente no estaba tan distante del desarrollo orientado hacia el mercado como algunos estereotipos sugieren. Bartolomé Yun ha mostrado que en muchos casos los ingresos de la aristocracia dependían fuertemente de alcabalas enajenadas. $\mathrm{Al}$ ser los réditos de la alcabala una función de la actividad de mercado, éste era un poderoso incentivo para que los terratenientes impulsaran una agricultura orientada hacia el mercado ${ }^{35}$. En la misma línea, Richard Herr ha mostrado que los poderosos locales adquirieron tierras en las desamortizaciones de Godoy como parte de una estrategia desarrollada para reorganizar los factores de producción en el sentido del mercado ${ }^{36}$.

Este modelo sorprendentemente dinámico de respuesta local a las posibilidades económicas tuvo lugar dentro de un sistema urbano que yo mismo caractericé una vez como estático y macrocefálico. Claro que era macrocefálico, dado que Madrid tenía 200.000 habitantes en 1800 y la siguiente ciudad en la red, como ya he definido, era Valladolid, que sólo contaba con unos 25.000 habitantes. En el nivel de las ciudades que llegaron a ser importantes en el siglo XVI la región era ciertamente estática. Ciudades como Cuenca, Ávila, Segovia y Toledo contaban con menos de 20.000 habitantes. Eran mucho menores que a finales del siglo XVI y apenas cambiaton entre 1750 y 1888 . Las únicas gtandes ciudades castellanas del siglo XVI que se recuperaron y expandieron en el XIX fueron Valladolid y Burgos, convirtiéndose ambas en importantes conexiones ferroviarias.

En realidad, si optamos por enfocar nuestra investigación hacia las redes de actividad en los mercados y el intercambio económico, el interior era una red tejida con poca fuerza, dentro de la cual la orientación hacia Madrid sobresalía. Sobresalía porque Madrid alcanzaba a gran parte de la región, no porque la dominase o integrase económicamente. Podemos observar mejor esta característica si tenemos en cuenta la prontitud con la que las áreas interiores respondieron a otros mercados cuando éstos aparecieron.

\section{MÁS ALLÁ DE CASTTLA: EL NORTE, LEVANTE Y ANDALUCIA}

Llegados a este punto sería útil ampliar nuestro enfoque y tratar de considerar toda la España peninsular, en lugar de limitarnos al interior de Castilla. Si sobreponemos las diferentes tendencias de conexión de redes de la aristocracia

34 RINGROSE, D., «An Approach to the Study of Market Integration in Nineteenth-Century Spainy, ponencia presentada en la Confenencia sobre Modernización Comparativa en el Mediterráneo, Sevilla, 1984.

35 YUN CASALILLA, B. Sobre la transición al capitalismo en Castilla: Economia y sociedad en Tierra de Campos (1500-1830), Salamanca, Junta de Castilla y León, 1987, págs. 314, 493; YUN CASALILLA, «Notas sobre el régimen señorial en Valladolid y el estado señotial de Medina de Rioseco en el siglo XVIIh", Investigaciones Históricas, vol. 3, 1982, págs. 145-176 y YUN CASALILLA, «Aristocracia, señonio y crecimiento económico en Castilla: Algunas reflexiones a partir de los Pimentel y los Enúquez (siglos XVI y XVII)", Revista de Historia Económica, vol. 3, n. 3,1985 , págs. 443-471.

36 HERR, R., Rural Change and Royal Finances in Spain at the End of the Old Regime, Berkeley, University of California Press, 1989 , paigs. 602-605. 
terrateniente, las elites burocráticas y la estructura administrativa del estado, los patrones de migración rural-urbano y el comercio y las finanzas en un solo mapa, es fácil diseñar el marco de una red urbana castellana del siglo XVIII que incluya la costa norte, el País Vasco y Andalucía. Ésta tendría una distribución rango-tamaño razonablemente regular y sus ciudades más grandes se encontrarían en el centro (Madrid) y en el sur (Sevilla, Cádiz, Málaga, Córdoba y Granada). También se podría incluir el Levante, aunque a finales del siglo XVIII Barcelona y Valencia podrían no haber encajado muy bien en el ideal de jerarquía necesatio para una red urbana bien integrada.

El problema de esta aproximación es que, mientras hasta cierto punto podría presentar una descripción adecuada, no explicaría mucho sobre qué era lo que realmente sucedía entre las diferentes regiones y ciudades del sistema. De hecho, podríamos sugerir que lo que aparecía como un orden rango-tamaño plausible para las grandes ciudades de un sistema concreto era en realidad el resultado accidental de mezclar elementos desconectados y obtener un resultado en apariencia aceptable. De nuevo nos vemos confrontados por la observación de de Vries de que uno de los problemas básicos al analizar redes urbanas es el establecimiento de los límites apropiados para aquello que está siendo observado. El problema es, de hecho, similar al que Sánchez León señala con respecto a la aproximación deductiva empleada por de Vries al tratar las ciudades europeas más pequeñas.

$\mathrm{Si}$, de cualquier manera, observamos los diversos tipos de actividades de conexión de redes implicadas, descubrimos que el único denominador común real que convierte a Madrid en el centro de un sistema peninsular es su jurisdicción regia. Independientemente de la intensidad o laxitud con la que esa jurisdicción era puesta en práctica, ésta alcanzaba todos los puntos del país, extrayendo recursos, haciendo uso de los fondos regios y administrando justicia y defensa. El comportamiento autónomo de la aristocracia terrateniente en relación con las redes a menudo actuaba de forma paralela a esa concentración en Madrid y de este modo canalizaba cantidades sustanciales de los ingresos rurales hacia esa economía urbana. De las grandes familias, muy pocas abandonaron completamente, ya sea personal o psicológicamente, sus bases provinciales de poder y riqueza. Esto es particularmente importante para llegar a comprender cómo Andalucía encaja en ese pátrón de regiones peninsulares. Considero una cuestión abierta si aquellos aristócratas que construín palacios en Madrid estaban necesariamente trasladándose a la capital, o si muchos de ellos estaban colonizando Madrid desde un universo andaluz que tenía su capital residencial en Sevilla. Algo similar podría argumentarse con respecto a la nobleza provincial, educada en la universidad, de Asturias y Cantabria. Al desempeñar un importante papel en la nobleza burocrática que dominaba el gobierno de los Borbones, ¿trasladaban éstos sus casas patrimoniales a Madrid en sentido permanente o colonizaban la burocracia desde esas casas patrimoniales provinciales?

Esta ambigüedad con respecto a la emigración a Madrid que podría haberse dado en el caso de los aristócratas y burócratas es todavía más intrigante en el caso de las familias relacionadas con los negocios y las finanzas. Una propor- 
ción extraordinariamente elevada de la comunidad banquera y de la comercial en Madrid no era sólo vasca o navarra sino que además había nacido en esas provincias. Esto puede afirmarse de muchas de las firmas familiates que mantuvieron casas y oficinas en la ciudad durante varias generaciones. Aquellas mismas familias comerciales de base provincial también enviaron miembros y afiliados hacia otros gtandes centros de mercado ${ }^{37}$. Así, comerciantes vascos fueron también importantes en el florecimiento de Santander y La Coruña como puertos comerciales. Las familias vascas y cántabras de comerciantes mantenían también compañías en Cádiz ${ }^{38}$. Como compañías gaditanas participaron en el comercio americano durante generaciones, usando parientes reclutados regularmente en sus pueblos de origen en el norte. Los comerciantes navarros fueron a Francia y Levante tan fácilmente como a Madrid ${ }^{39}$. Tal y como los empresarios catalanes entraron también en otras partes de la economía peninsular del siglo XVIII, ya fuera en Málaga, Madrid o La Coruña, ésta era una manera clara de establecer dependencias de la casa patrimonial. Inevitablemente Madrid era un importante centro para los negocios, pero, como mercado final no era necesariamente la residencia del eje central de la empresa familiat.

Esta aproximación a las redes nos permite reconocer que Madrid era el centro de muchas cosas en España, y el resultado puede ser descrito ciertamente como una jerarquía peninsular de lugares centrales. Al mismo tiempo también es legítimo observar por separado los estratos individuales de la conexión de redes. Si hacemos esto y nos fijamos en los patrones de interconexión generados por gente que responde a una oportunidad comercial, obtenemos pautas regionales distintivas, modelos que arrojan algo de luz sobre la situación de mercado apatentemente descoordinada que se describe para el interior de Castilla en páginas anteriores.

Como ya he tratado de explicar en anteriores ocasiones, España en los siglos XVI y XVII desarrolló cuatro modelos regionales de actividades comerciales o mercantiles, de los cuales el más nebuloso y debatible era el del interior de Castilla, que he discutido más arriba. Por defecto, éste parece estar orientado hacia Madrid, pero en un examen mas detallado se aprecia que su modesta actividad mercantil respondía a una dispersa colección de incentivos. El más cla-

37 CRUZ VALENCIANO, J., "Cambistas madrileños de la segunda mitad del siglo XVIII", en Otero CarvajAL, L. E. y BAHAMONDE, A. (eds.), Madrid en la sociedad del siglo XIX, vol.I: La cithdad y su entorno; Madrid centro de poder politico; Poder económico y élites locales, Madrid, Comunidad de Madrid, 1986, págs. 453-476; RINGROSE, D., «Hombres de negocios y hombres de la politica, fines del siglo XVIII y principios del XIX", Actar del segundo congreso sobre archivos económicos de entitades privadas, Madrid, Banco de España, 1988, págs. 65-76.

38 RuIz RIVERA, J. B., El Consulado de Cádir: Matricula de comerciantes (1730- 1823), Cádiz, Diputación Provincial, 1988.

39 Caro Baroja, J., La bora navarra (Personas, familias, negacios e ideas), Pamplona, Comunidad Foral de Navarra, s.d. Más recientementr. esta conexión de redes en sociedades regionales ha sido documentada en AZCONA GUERRA, A. M., Comercio y comerciantes en la Navarra del siglo XVIII, Pamplona, Gobierno de Navarra, 1996, págs. 65-92.

Hi.ppantia, LVIIL/2, núm. 199 (1998) 489-512 
to y el más antiguo sistema urbano peninsular tiene su base en Barcelona. Independientemente de sus actividades medievales, que se extendieron hasta alcanzar Túnez, Alejandría y Atenas, en el siglo XVI Barcelona formaba parte de un sistema multicéfalo en el Mediterráneo occidental. Esta serie de ciudades interdependientes incluía Génova, Nápoles, Palermo, Livorno y —posiblemente-- Marsella y mantenía un contacto estable con Amberes y los mercados noteuropeos coordinados a través de este puerto. Durante el siglo XVII Barcelona pasó de 30.000 a 100.000 habitantes y se convirtió en uno de los centros de coordinación dominantes de cara al Mediterráneo occidental. En España, su influencia se extendía hacia el interior hasta Zaragoza y se expandía hacia el sur hasta Alicante. Mientras los mercaderes catalanes penetraban en el comercio atlántico después de 1740, Málaga fue también, durante un tiempo, incluida en su sistema comercial. Es en este contexto en el que vemos a los mercaderes catalanes aparecer en Madrid a finales del siglo XVIII, buscando vías de acceso a este mercado.

La tercera red urbana bien establecida era la de Andalucía, particularmente la del valle del Guadalquivir. La lógica económica de Ia región teflejaba la producción de trigo, lana, vino y aceite de oliva orientada hacia el mercado y con partidas cada vez mayores destinadas a la exportación. Dependiendo de las circunstancias, las salidas comerciales para esta actividad se encontraban en Cádiz o en Sevilla y, aparte de la exportación, estas dos ciudades constituían un mercado urbano comparable al de Madrid. Las fronteras entre aquella Andalucía y las hoy ciudades andaluzas de Granada y Málaga eran extremadamente permeables. Hasta qué punto estas dos ciudades estaban ligadas a Andalucía o a Levante depende del tipo de interacciones interurbanas o rural-urbanas que pretendamos resaltar, así como del período que elijamos. La cuenca del Guadalquivir puede definitse tanto en términos de conexiones de mercado que se concentran en Sevilla y Cádiz, como de hábitos residenciales de las elites acaudaladas de la región, en lo cual destaca Sevilla.

La cuarta red que se define comercialmente, que no emerge hasta el siglo XVIII, es la de la costa norte. El centro de este sistema lo forman Bilbao y el País Vasco con un comercio de importación estable de Bilbao a Madrid, y su función exportadora de la lana castellana. En un ataque indirecto de los privilegios aduaneros de la región vasca, la Corona comenzó en la década de 1750 a construir caminos y canales destinados a reorientar el comercio castellano hacia Santander. Durante la década de 1760, la Corona seleccionó a La Coruña como puerto para su correo marítimo con fragatas ligeras que llevaban carga y pasajeros a la Habana y a Buenos Aires. Posteriormente, ambos puettos fueron autorizados para el comercio directo con América, mientras que Bilbao fue excluido de una participación directa. Estas políticas impulsaron el desplazamiento de los mercaderes y el capital vasco y cántabro hacia Santander y Galicia. Las últimas décadas del siglo XVIII vieron, de este modo, el florecimiento de una red comercial interurbana a lo largo de toda la costa norte, que legaría a ser muy importante en el siglo XIX. En 1800 todavía se mantenía tenue y sus ciudades clave eran minúsculas en comparación con las de Andalucía

Hispania, LVIII/2, nưm. 199 (1998) 489-512 
o Levante ${ }^{40}$. Aun en estas circunstancias, esta red estaba suficientemente establecida como para sobrevivir a la pérdida del apoyo de la administración después de 1800.

Las tres redes regionales de actividades mercantiles en la periferia, que ayudaron a crear y se beneficiaron de redes y jerarquías urbanas y regionales, nos permiten entender la naturaleza dispersa de las redes mercantiles en Castilla. Mientras que Madrid atraía mercancías desde la mayor parte del área comprendida entre la Cordillera Cantábrica y Sierra Morena, el carácter fluido de los mercados, del transporte y de la producción hacian que el interior de Castilla fuera susceptible de colonización por las actividades mercantiles generadas por las tres redes urbanas de la costa. El ganado vacuno gallego hasta entonces vendido en Madrid, empezó a ser exportado a Inglaterra a partit de 1814. La lana fue siempre una de las exportaciones principales, pero la demanda de lana más barata producida localmente varió significativamente en relación a la demanda de lana de merino trashumante. La producción maderera de la provincia de Soria que había abastecido habitualmente a Madrid fue desviada gradualmente hacia los mercados de los puertos del norte. Castilla la Vieja, que históricamente había vendido su trigo en las ciudades locales y en Madrid, comenzó a exportarlo, junto con la harina, a través de Santander. Ya en la década de 1750 cantidades significativas de trigo manchego eran exportadas desde Alicante hacia Barcelona. En el sur, el comercio andaluz no sólo incluía el mercurio de las minas de Almadén con sus consiguientes demandas de madera y combustible, sino también la lana y la ganadería procedente de Extremadura. A finales del siglo XVIII los mercaderes andaluces pugnaban no sólo con la competencia de Madrid sino también con la de Valencia.

\section{CONCLUSIÓN}

Para finalizar, quiero reiterar las apreciaciones de Sánchez León, al señalar la importancia de considerar los factores institucionales en las explicaciones de la urbanización, y al demostrar que no es posible sustituir con facilidad deducciones por datos empíricos. Al mismo tiempo, al ignorar la posibilidad de que los mercados integrados jugaran un importante papel en la configuración del sistema urbano de la Castilla del siglo XVI, renunciamos al valor heurístico de caracterizar las ciudades en términos de redes y jerarquías urbanas. En efecto, las instituciones y los mercados interactúan. Sin ellos es imposible entender las redes urbanas españolas del siglo XVIII.

Además, esta nueva noción de la importancia de los factores sociales e institucionales y de la naturaleza de las pequeñas ciudades castellanas nos permite una importante reorientación de algunas de las cuestiones más elementales sobre siglo XVII. En lugar de tratar de explicar el declive de la urbanización caste-

40 Mientras Barcelona tenía 100.000 habitantes en 1800 , Valencia 75.000 , y Málaga 50.000 , Bilbao tenía sólo 11.000, La Coruña 10.000, y Santander apenas 5.000. 
llana, como muchos hemos intentado, podemos ahora hablar en términos de redistribución de funciones urbanas. La mayor parte de la vida urbana castellana, basada en los hábitos residenciales de las elites locales y en la variedad de posibilidades dentro de los mercados locales señalada por Epstein, persiste a lo largo de todo el siglo XVII. Esto, en consecuencia, ayuda a explicar algunas evoluciones aparentemente aleatorias del siglo XVIII. Más allá de la sombra macrocefálica de un Madrid subsidiado políticamente, podemos apreciar que el campo castellano estaba provisto de núcleos dispersos de consumo de elite y de servicios urbanos. Esto hizo posible la respuesta de los productores a las oportunidades del metcado que llegaron al interior desde la periferia. Las condiciones institucionales locales podrían o no haber estimulado estas respuestas, e indudablemente determinaban qué oportunidades de fuera obtenían respuesta, pero el campo castellano estaba más capacitado para responder de lo que siempre hemos creído.

Pot esto, un acercamiento a la urbanización que reconoce la existencia de las ciudades dentro de un entorno compuesto por diversos tipos de interacción, cada uno con sus propios imperativos locales, es importante si queremos que la historia urbana vaya más allá de la mera descripción de sistemas de lugares centrales y la comparación de distribuciones de rango-tamaño. Combinando estas herramientas con la conciencia de que las conexiones en cualquier red son creadas por individuos que toman decisiones basadas en motivos complejos, quizás podremos entender mejor por qué las ciudades y las redes urbanas funcionan como lo hacen en sus contextos históricos.

Traducción de Alejandro Díaz Bueno y Raquel Romero 